\title{
Therapie des Antiphosphoplidsyndrom (APS) mit DOAK
}

\author{
Stellungnahme der wissenschaftlichen Fachgesellschaften GTH, DGA, DGP sowie von
} BDDH und des Aktionsbündnis Thrombose zum Rote Hand Brief vom 23.05.2019

\section{Treatment of the antiphospholipid syndrome with direct oral anticoagulants}

\author{
Position statement of the scientific societies Society of Thrombosis and Haemostasis \\ Research, German Society of Vascular Medicine, German Society of Phlebology, \\ Berufsverband der Deutschen Hämostaseologen, German League of Vascular \\ Diseases and the Action Alliance Thrombosis
}

Autoren

R. Bauersachs ${ }^{1,2}$, S. Schellong ${ }^{3}$, M. Stücker ${ }^{4}$, J. Oldenburg ${ }^{5}$, C. Kalka ${ }^{6}$, U. Scholz ${ }^{7}$, E. Lindhoff-Last ${ }^{8}$

Institute

1 Klinik für Gefäßmedizin- Angiologie, Klinikum Darmstadt $\mathrm{GmbH}$

2 Center of Thrombosis and Hemostasis, Universitätsmedizin Mainz, Mainz

3 Städtisches Klinikum Dresden, Medizinische Klinik 2

4 Klinik für Dermatologie, Venerologie und Allergologie, Ruhr-Universität Bochum

5 Institut für Experimentelle Hämatologie und Transfusionsmedizin, Universitätsklinikum Bonn

6 Marienhospital Brühl GmbH, Innere Medizin 1

7 Center of Hemostasis Leipzig

8 CardioAngiologisches Centrum Bethanien (CCB), CCB-Gefäßzentrum und CCB-Gerinnungszentrum, Frankfurt

\section{Schlüsselwörter}

Antiphospholipid-Syndrom, Antikoagulation, Vitamin K Antagonisten, Direkte orale Antikoagulanzien, Lupus Antikoagulans, Anti-Phospholipid-Antikörper

Key words

Antiphospholipid-Syndrome, Anticoagulation, Vitamin K Antagonists, Direct oral anticoagulants, Lupus Anticoagulant, Anti-Phospholipid-antibodies

eingereicht 26.06.2019

akzeptiert 26.06.2019

Bibliografie

DOI https://doi.org/10.1055/a-0962-5481

Phlebologie 2019; 48: 271-274

(c) Georg Thieme Verlag KG Stuttgart · New York ISSN 0939-978X
Korrespondenzadresse

Prof. Dr. med. Rupert Bauersachs

Klinik für Gefäßmedizin - Angiologie,

Klinikum Darmstadt GmbH

E-Mail: rupert.bauersachs@mail.klinikum-darmstadt.de

\section{ZUSAMMENFASSUNG}

Das Antiphospholipid-Syndrom ist eine der schwerwiegendsten thrombophilen Störungen, die nicht nur zu rezidivierenden venösen, sondern auch zu arteriellen Thromboembolien sowie Schwangerschaftskomplikationen führen kann. Zusammen mit dem klinischen Bild ist das APS durch spezifische Laborbefunde charakterisiert: 1. Lupus Antikoagulans (LA), 2. AnticardiolipinAntikörper (ACA), 3. $\beta 2$-Glykoprotein I-Antikörper (B2GPI-AK). Alle Testergebnisse müssen nach 12 Wochen bestätigt werden. Sind alle drei Testgruppen positiv, besteht das höchste thrombotische Risiko. Beachtet werden muss, dass LA-Tests unter UFH, VKA oder DOAKs falsch positiv ausfallen können; bei DOAKs scheint die Zugabe von Absorbern nach Blutentnahme zuverlässige Ergebnisse zu liefern.

Eine Vergleichsstudie (TRAPS) zwischen VKA und dem DOAK Rivaroxaban mit ausschliesslich 3-fach positiven HochrisikoPatienten wurde vorzeitig abgebrochen, wegen erhöhter Ereignisraten unter Rivaroxaban [19\% zumeist arterielle Ereignisse versus $3 \%$ unter Warfarin (HR 7.4; 1.7-32.9)]. Ein daraufhin herausgegebener Rote-Hand-Brief warnt vor der Anwendung von DOAKs bei Patienten mit APS, insbesondere bei HochRisiko- (3-fach positiven) Patienten, und empfiehlt die Überprüfung einer laufenden DOAK-Therapie und eine mögliche Umstellung auf VKA, insbesondere bei Hoch-Risiko-Patienten. Als Fazit soll 1. bei klinischem Verdacht eine sorgfältige APSDiagnostik erfolgen. Viele Patienten haben aufgrund von inadäquater Diagnostik wahrscheinlich gar kein APS und können bei venöser Thromboembolie adäquat ein DOAK erhalten. 
2. Bei einfach- oder zweifach-positiven Antiphospholipid-AK Tests ohne LA besteht ein vergleichbar niedriges Thromboserisiko; auch hier kann möglicherweise ebenfalls mit DOAKs behandelt werden, wenn venöse Thrombosen vorliegen - ausreichende Daten liegen noch nicht vor, aber Metaanalysen legen dies nahe. 3. Patienten mit Positivität in allen 3 APS-Tests und APS-Patienten mit arteriellen Thromboembolien haben ein sehr hohes Risiko. Die TRAPS-Studie zeigt, dass diese Patienten nicht mit DOAKs, sondern mit einem VKA behandelt werden sollen.

\section{ABSTRACT}

The antiphospholipid-syndrome (APS) is one of the most severe forms of thrombophilia, which may not only lead to recurrent venous but also to arterial thromboembolic events (TE), and to severe pregnancy complications, respectively. Together with clinical symptoms APS is defined by specific laboratory findings: 1. Lupus anticoagulant (LA), 2. anticardiolipin-antibodies $(A C A)$, and 3. $\beta 2$-Glycoprotein I-antibodies ( $\beta 2 \mathrm{GPI}-\mathrm{Ab}$ ). All test results have to be confirmed after at least 12 weeks. The thrombotic risk is highest, if all 3 test groups are positive. It must be pointed out that the presence of UFH, VKA or DOACs may lead to false positive LA-test results; the addition of a specific absorber after blood sampling may provide reliable results in the presence of DOACs.

A prospective randomized controlled trial comparing warfarin and rivaroxaban (TRAPS-trial) including high-risk patients only with triple positive APS was terminated early because of an increased rate of TE in patients treated with rivaroxaban [19\%, mostly arterial, compared to $3 \%$ with warfarin (HR 7.4;1.732.9)]. Subsequently, a warning letter was issued by the pharmaceutical manufacturers of DOACs, including a warning of DOAC use in APS-patients, particularly in triple-positive high risk patients.

Conclusions: 1 . Clinical suspicion of APS requires careful diagnostic testing. Because of inadequate diagnostic workup, many patients may not even have an APS, and these patients could be adequately treated with a DOAC. 2. Patients with single or double positive antiphospholipid antibodies but without positive LA may have a comparably low thrombotic risk and may also be treated with a DOAC in venous TE - sufficient evidence for that conclusion is not yet available, but is suggested by the results of meta-analyses. 3. Triple positive patients or those with APS who suffered from arterial thromboembolism have a very high recurrence risk of thrombosis; The TRAPS-Study shows, that these patients should be treated with VKA instead of a DOAC.

\section{Das Antiphospholipidsyndrom (APS)}

Unter den thrombophilen Diathesen stellt das APS die größte Herausforderung dar, da es mit einem sehr hohen thrombotischen Risiko einhergehen kann. Hauptmanifestationen sind - überwiegend bei jungen Patienten - arterielle und venöse Thrombosen sowie Schwangerschaftskomplikationen (1). Häufig bestehen gleichzeitig Autoimmunerkrankungen wie z. B. ein systemischer Lupus erythematodes (sekundäres Antiphospholipid-Syndrom). Ein erheblicher Teil der Patient*innen hat aber unprovozierte Thromboembolien oder Schwangerschaftskomplikationen ohne eine zugrundeliegende Grunderkrankung (primäres Antiphospholipid-Syndrom).

\section{Diagnostik}

Zusammen mit diesem klinischen Bild ist das APS durch Laborbefunde definiert, nämlich: positive Lupusantikoagulantien (LA), Anticardiolipin-Antikörpern (ACA, IgG oder lgM) und/oder $\beta 2-G l y-$ koprotein I-Antikörper ( $\beta 2 \mathrm{GPI}$-AK, IgG oder IgM). Relevant sind diese Antikörper nur bei wiederholt positivem Nachweis mindestens 12 Wochen nach der erstmaligen Bestimmung. Aktuelle APSKlassifikationskriterien wurden bei der Sapporo-Konsensuskonferenz mit Sydney-Revision erarbeitet (2). Das Scientific Standardisation Subcommittee (SSC) der ISTH hat folgende Laborkriterien für das APS herausgegeben (3): Bei dreifach-positiven Laborbefunden sind alle drei Messwerte (LA, ACA und 32 GPI-AK) positiv, was mit dem höchsten Thromboserisiko einhergeht. Doppelt positive APSPatienten (meist LA negativ) weisen grundsätzlich ein niedrigeres Thromboserisiko auf, einfach positive Patienten (bezogen auf ACA und $\beta 2-\mathrm{GPI}-\mathrm{AK}$ ) haben das geringste Risiko. Unbedingt muss beach- tet werden, dass der DRVVT-Test zum Nachweis von LA sowohl bei Therapie mit DOAK als auch bei VKA oder UFH verfälscht werden kann, so dass der Test falsch positiv ausfällt und irrtümlicherweise angenommen wird, dass, Lupusantikoagulantien vorliegen $(4,5)$. Die korrekte Labordiagnostik bei bereits oral antikoagulierten Patienten erfordert daher eine Therapiepause bis zum vollständigen Abklingen der Gerinnungshemmung. Bei sehr hohem Thromboserisiko sollte die Pause durch niedermolekulares Heparin überbrückt werden. Auch ohne DOAK-Pause scheint die Zugabe von speziellen Absorbern in das Blutentnahmeröhrchen für die Testung von LA zuverlässige Ergebnisse zu liefern (6).

\section{Therapie}

In der Therapie entscheidend ist die Antikoagulation. Der bisherige Standard sind VKA. Niedrigdosiertes ASS kann als Monotherapie in der primären Thromboseprophylaxe des APS oder in Kombination mit oraler Antikoagulation insbesondere bei arteriellen Thrombosen und gleichzeitig vorliegendem vaskulären Risikoprofil eingesetzt werden (7). Direkte orale Antikoagulantien (DOAKs) wie Dabigatran, Rivaroxaban, Apixaban oder Edoxaban werden auf Grund ihrer vorhersagbaren Wirkung ohne Monitoring und ihrem guten Risiko/Nutzenprofil zunehmend in der Therapie des APS eingesetzt. Insbesondere bei APS-Patienten mit starkem LA, bei denen ein verlässliches INR-Monitoring unter VKA-Therapie erschwert sein kann, können DOAKs Vorteile bezüglich einer verlässlichen Antikoagulation bieten. Eine datenbasierte Evidenz zu ihrem Einsatz fehlte aber bisher. 


\begin{tabular}{|c|c|c|c|}
\hline $\begin{array}{l}\text { Schweregrad des Antiphospholipid- } \\
\text { Syndroms }\end{array}$ & $\begin{array}{l}\text { DOAK-Therapie } \\
\text { (nur therapeutische Dosierungen) }\end{array}$ & VKA-Therapie & VKA-Therapie mit ASS-Therapie \\
\hline APS mit arteriellen Thrombosen & Kein Einsatz von DOAK & Goldstandard VKA & $\begin{array}{l}\text { Bei vaskulären Risikofaktoren } \\
\text { zusätzlich ASS erwägen }\end{array}$ \\
\hline $\begin{array}{l}\text { Schweres APS mit dreifach positiven Anti- } \\
\text { phospholipid-Tests und venösen Thrombosen } \\
\text { (LA und ACA-AK und } \beta 2 \text {-GPI-AK) }\end{array}$ & Kein Einsatz von DOAK & Goldstandard VKA & Nicht erforderlich \\
\hline $\begin{array}{l}\text { Moderates APS mit zweifacher Antikörper- } \\
\text { positivität, negativem LA und venösen } \\
\text { Thrombosen (ACA-AK und } \beta 2-G P I-A K)^{*}\end{array}$ & Einsatz von DOAK möglich & Alternativ VKA & Nicht erforderlich \\
\hline $\begin{array}{l}\text { Mildes APS mit einfacher Antikörperpositivität, } \\
\text { negativem LA und venösen Thrombosen } \\
\text { (ACA-AK oder } \beta 2 \text {-GPI-AK)* }\end{array}$ & Einsatz von DOAK möglich & Alternativ VKA & Nicht erforderlich \\
\hline Kein APS (z.B. falsch positive LA) & Einsatz von DOAK & Alternativ VKA & Nicht erforderlich \\
\hline
\end{tabular}

\section{Neue Daten zur Antikoagulation bei APS}

Kürzlich wurde erstmals ein Vergleich zwischen VKA und dem DOAK Rivaroxaban bei der Therapie des APS publiziert. In der TRAPS-Studie (Trial on Rivaroxaban in AntiPhospholipid Syndrome), einer randomisierten, offenen, multizentrischen Nicht-Unterlegenheitsstudie mit verblindeter Endpunkt-Adjudizierung, wurde die Wirksamkeit und Sicherheit von Rivaroxaban $1 \times 20 \mathrm{mg}$ ( $1 \times 15 \mathrm{mg}$ bei eingeschränkter Nierenfunktion) mit Warfarin (INR Ziel 2.0-3.0) bei Patienten mit thrombotischem APS verglichen (8). Nur Hochrisikopatienten mit dreifacher Positivität wurden in die Studie aufgenommen. Die Studie wurde nach Einschluss von 120 Patienten (59 Rivaroxaban, 61 Warfarin) wegen erhöhter Ereignisraten unter Rivaroxaban vorzeitig abgebrochen. Nach einem mittleren Follow-up von 569 Tagen kam es zu 19\%, zumeist arteriellen Ereignissen im Rivaroxabanarm und zu $3 \%$ Ereignissen in der Warfarin Gruppe (HR 7.4; $95 \%$ KI1.7-32.9). Schwere Blutungen traten bei sechs Patienten auf: 4 (7\%) unter Rivaroxaban und 2 (3\%) unter Warfarin (HR 2.3; $95 \%$ KI 0.4-12.5). Es wurden keine Todesfälle dokumentiert (8).

Eine aktuelle internationale Metaanalyse mit individuellen Patientendaten (9) wertete 447 APS Patienten aus, die mit Rivaroxaban ( $n=290)$, Dabigatran (=144) und Apixaban $(n=13)$ behandelt worden waren. Auch hier ergaben sich während einer mittleren Beobachtungszeit von 12,5 Monaten hohe Rezidivthromboseraten von $16 \%$. Ein signifikant erhöhtes Rezidivrisiko hatten dreifach-positive Patienten [4-fach erhöht ( $56 \%$ vs. $23 \%$; OR = 4,3; $95 \% \mathrm{KI} 2,3-$ $7,7 ; p<0,0001)]$, und Patienten mit vorausgegangenem arteriellen Ereignis oder small-vessel-Erkrankung. Auch diese Studie zeigt, dass DOAKs nicht bei allen APS-Patienten effektiv sind (9). Dagegen finden sich Hinweise dafür, dass DOAKs bei APS-Patienten mit venösen Thromboembolien und einem niedrigen Thromboembolierisiko eine wirksame und sichere Alternative zu VKAs darstellen (10).

\section{Rote-Hand Brief}

Aufgrund der Ergebnissen der TRAPS-Studie wurde am 23.5.2019 ein Rote Hand Brief herausgegeben (https://www.bfarm.de/Sha-
redDocs/Risikoinformationen/Pharmakovigilanz/DE/RHB/2019/ rhb-doaks.html), mit dem Warnhinweis „die Anwendung von DOAKs wird bei Patienten mit APS nicht empfohlen, besonders bei Hoch-Risiko-Patienten (Patienten, die in allen drei Antiphospholipid-Tests positiv getestet wurden - Lupus-Antikoagulans, Anti-Cardiolipin-Antikörper und Anti-Beta-2-Glykoprotein-I-Antikörper).“ sowie der Empfehlung: „Überprüfen Sie, ob bei Patienten mit APS, die zurzeit mit DOAKs zur Prävention thromboembolischer Ereignisse behandelt werden, eine Fortsetzung der Behandlung angemessen ist und erwägen Sie eine Umstellung auf Vitamin-KAntagonisten. Dies gilt insbesondere für Hoch-Risiko-Patienten.“

\section{FAZIT FÜR DIE PRAXIS}

Als Fazit für die Praxis ergeben sich hieraus mehrere Konsequenzen:

1. Die Durchführung einer APS-Diagnostik setzt eine klare klinische Fragestellung voraus. Bei klinischem Verdacht auf ein APS ist eine sorgfältige, objektive Diagnostik in spezialisierten Labors zur Risikostratifizierung unverzichtbar. Viele Patienten - möglicherweise sogar die Mehrheit, die als APS klassifiziert werden - haben wahrscheinlich diese Erkrankung gar nicht, da die Labordiagnostik nicht adäquat durchgeführt wurde (siehe oben). Diese Patienten - die tatsächlich gar kein APS haben -stellen daher eine Niedrigrisikogruppe für Rezidiv-VTE dar, deren Ereignis von venöser Thromboembolie adäquat mit einem DOAK therapiert werden kann $(1,2)$.

2. Patienten mit einfacher oder zweifacher Antiphospholipid-Antikörperpositivität ohne positiven Nachweis von Lupusantikoagulantien (ACA und/oder B2GPI-AK-Positivität) haben ein niedriges Thromboserisiko und könnten möglicherweise ebenfalls mit DOAK behandelt werden, wenn venöse Thrombosen vorliegen. Es muss aber betont werden, dass für dieses Patientenkollektiv bisher keine Daten zur Behandlung mit DOAKs vorhanden sind. Die 
Metaanalyse von 2019 (10) kann aber als Hinweis für diese Schlussfolgerung dienen.

3. Patienten mit einem Hochrisiko-APS mit Positivität in allen 3 APS-Tests und APS-Patienten mit arteriellen Thromboembolien haben dagegen ein sehr hohes Risiko für thrombotische Komplikationen, und die TRAPS-Studie (8) zeigt, dass diese Patienten nicht mit DOAKs, sondern nur mit einem VKA (im Falle der Studie Warfarin) im Zielkorridor INR 2,0-3,0 behandelt werden sollen. Ob bei Patienten mit vorausgegangenen arteriellen Thrombosen zusätzlich ASS eingesetzt werden soll, ist bisher unklar, wird aber durch die TRAPS-Studie unterstützt. Die TRAPS-Studie bestätigt den Einsatz von VKAs (INR 2,0-3,0) als Standardtherapie bei Hochrisiko-APS mit dreifacher Positivität (1), der nicht zugunsten von DOAKs verlassen werden darf.

\section{Potenzielle Interessenkonflikte}

R. Bauersachs: Studienleitung Aspen, Bayer, BMS, Leo; Honoraria von Aspen, Bayer, Boehringer, BMS, Daiichi Sankyo, Leo und Pfizer S. Schellong: Forschungsunterstützung von BMS; Honoraria von Aspen, Bayer, Boehringer, BMS, Daiichi Sankyo und Pfizer M. Stücker: Beraterhonorar Bayer; Forschungsunterstützung: Daiichi Sankyo

J. Oldenburg: Forschungsunterstützung, Beratungs- und Vortragshonoraria, sowie Unterstützung von Kongressteilnahme von Bayer, Pfizer und Roche.

C. Kalka: Vortrags- und Beratungshonoraria von BMS/Pfizer und Berlin-Chemie

U. Scholz: Keine

E. Lindhoff-Last: Forschungsunterstützung, Vortrags- und Beratertätigkeit: Aspen, Bayer, BMS/Pfizer, Boehringer-Ingelheim, Daiichi-Sankyo, CSL Behring

F. Langer: Honorare von Bayer, BMS/Pfizer, Boehringer, Daiichi Sankyo R. Klamroth: Forschungsunterstützung und Honorare von Bayer, BMS, Daiichi Sankyo, Pfizer

\section{Literatur}

[1] Bauersachs R. Antikoagulation bei venösen und arteriellen Thromboembolien. In: Lindhoff-Last E, Kemkes-Matthes B, Oldenburg J, Tiede A (Hrsg.) Handbuch Hämostaseologie/Hämophilie. Wiesbaden: med publico $\mathrm{GmbH} ; 2$ 2018; 1-38

[2] Miyakis S, Lockshin MD, Atsumi T et al. International consensus statement on an update of the classification criteria for definite antiphospholipid syndrome (APS). J Thromb Haemost 2006; 4: 295-306

[3] Devreese KM], Ortel TL, Pengo $V$ et al. Subcommittee on Lupus Anticoagulant/Antiphospholipid A: Laboratory criteria for antiphospholipid syndrome: communication from the SSC of the ISTH. J Thromb Haemost 2018; 16: 809-813

[4] Linnemann B. Antiphospholipid syndrome - an update. Vasa 2018; 47: 451-464

[5] Garcia D, Erkan D. Diagnosis and Management of the Antiphospholipid Syndrome. N Engl J Med 2018; 378: 2010-2021

[6] Exner T, Michalopoulos N, Pearce J et al. Simple method for removing DOACs from plasma samples. Thromb Res 2018; 163: 117-122

[7] Pengo V, Denas G. Diagnostics and treatment of thrombotic antiphospholipid syndrome (APS): A personal perspective. Thromb Res 2018; 169: $35-40$

[8] Pengo V, Denas G, Zoppellaro G et al. Rivaroxaban vs warfarin in high-risk patients with antiphospholipid syndrome. Blood 2018; 132: 1365-1371

[9] Dufrost V, Risse J, Reshetnyak T et al. Increased risk of thrombosis in antiphospholipid syndrome patients treated with direct oral anticoagulants. Results from an international patient-level data meta-analysis. Autoimmun Rev 2018; 17: 1011-1021

[10] Elsebaie MAT, van Es N, Langston A et al. Direct oral anticoagulants in patients with venous thromboembolism and thrombophilia: a systematic review and meta-analysis. J Thromb Haemost 2019; 17: 645-656

[11] Laureano M, Crowther MA. Higher-risk APS: do we dare to DOAC? Blood 2018; 132: 1357-1358 